\title{
The Interaction Between Microglia and Macroglia in Glaucoma
}

\author{
Xiaohuan Zhao'tt, Rou Sun ${ }^{2 t}$, Xueting Luo ${ }^{1}$, Feng Wang ${ }^{3 *}$ and Xiaodong Sun ${ }^{1 *}$ \\ ${ }^{1}$ Shanghai Key Laboratory of Ocular Fundus Diseases, Shanghai General Hospital, Shanghai Engineering Center for Visual \\ Science and Photomedicine, Shanghai, China, ${ }^{2}$ Department of Ophthalmology, Ninth People's Hospital, Shanghai Jiao Tong \\ University School of Medicine, Shanghai, China, ${ }^{3}$ Department of Immunology and Microbiology, Shanghai General Hospital, \\ The Center for Microbiota and Immunological Diseases, Shanghai Institute of Immunology, Shanghai Jiao Tong University \\ School of Medicine, Shanghai, China
}

\section{OPEN ACCESS}

Edited by:

Eleonora Vannini,

National Research Council, Consiglio Nazionale delle Ricerche (CNR), Italy

Reviewed by:

Sabrina Reinehr,

University Eye Clinic, Ruhr-University

Bochum, Germany

Veit Rothhammer,

University Hospital FAU Erlangen

Nuernberg, Germany

*Correspondence:

Feng Wang

wangfeng16@sjtu.edu.cn

Xiaodong Sun

xdsun@sjtu.edu.cn

${ }^{\dagger}$ These authors have contributed equally to this work

Specialty section: This article was submitted to Neurodegeneration,

a section of the journal

Frontiers in Neuroscience

Received: 27 September 2020

Accepted: 22 April 2021

Published: 28 May 2021

Citation:

Zhao X, Sun R, Luo X, Wang F and Sun $X$ (2021) The Interaction Between Microglia and Macroglia

in Glaucoma.

Front. Neurosci. 15:610788. doi: 10.3389/fnins.2021.610788
Glaucoma, a neurodegenerative disease that leads to irreversible vision loss, is characterized by progressive loss of retinal ganglion cells (RGCs) and optic axons. To date, elevated intraocular pressure (IOP) has been recognized as the main phenotypic factor associated with glaucoma. However, some patients with normal IOP also have glaucomatous visual impairment and RGC loss. Unfortunately, the underlying mechanisms behind such cases remain unclear. Recent studies have suggested that retinal glia play significant roles in the initiation and progression of glaucoma. Multiple types of glial cells are activated in glaucoma. Microglia, for example, act as critical mediators that orchestrate the progression of neuroinflammation through proinflammatory cytokines. In contrast, macroglia (astrocytes and Müller cells) participate in retinal inflammatory responses as modulators and contribute to neuroprotection through the secretion of neurotrophic factors. Notably, research results have indicated that intricate interactions between microglia and macroglia might provide potential therapeutic targets for the prevention and treatment of glaucoma. In this review, we examine the specific roles of microglia and macroglia in open-angle glaucoma, including glaucoma in animal models, and analyze the interaction between these two cell types. In addition, we discuss potential treatment options based on the relationship between glial cells and neurons.

Keywords: microglia, macroglia, astrocytes, Müller cells, glaucoma, neuroinflammation

\section{BACKGROUND}

Glaucoma is one of the main causes of irreversible vision and visual field loss. As a progressive optic nerve disorder, its main pathological manifestations are loss of retinal ganglion cells (RGCs) and degenerative lesions of the optic nerve (Weinreb et al., 2014; Gordon and Kass, 2018; Calkins, 2021). Elevated intraocular pressure (IOP) has been recognized as a key risk factor for glaucoma.

Abbreviations: ATP, adenosine triphosphate; bFGF, basic fibroblast growth factor; CNTF, ciliary neurotrophic factor; Cx3cr1, C-X3-C motif chemokine receptor 1; C3ar1, C3a receptor 1; EAE, experimental autoimmune encephalomyelitis; EAG, experimental autoimmune glaucoma; GDNF, glial-derived neurotrophic factor; GFAP, glial fibrillary acidic protein; GLAST, glutamate/aspartate transporter; IL-1 $\beta$, interleukin $1 \beta$; IL-6, interleukin 6; IOP, intraocular pressure; LC, lamina cribrosa; LIF, leukemia inhibitory factor; MiRNAs, microRNAs; NF- $\mathrm{KB}$, nuclear factor kappa-B; ONA, optic nerve antigen; ONH, optic nerve head; RGCs, retinal ganglion cells; TANK, TNF receptor-associated factor NF- $\kappa B$ activator; TBK1, TANKbinding kinase 1 ; TGF- $\beta$, transforming growth factor- $\beta$; TGF- $\beta 2$, transforming growth factor- $\beta 2$;TLR, Toll-like receptor; $\mathrm{TNF} \alpha$, tumor necrosis factor $\alpha$; TSPO, translocator protein. 
Therefore, the main prevention and treatment measure for hightension glaucoma is adequate control of IOP (Weinreb and Khaw, 2004; Weinreb et al., 2014; Conlon et al., 2017; Gordon and Kass, 2018). However, some patients with normal IOP also have glaucomatous visual impairment and RGC loss. This suggests that in addition to high IOP, there may be other factors that cause pathological glaucomatous changes. For example, the duplication of TBK1 [tumor necrosis factor (TNF) receptorassociated nuclear factor kappa-B (NF- $\kappa \mathrm{B}$ ) activator (TANK)binding kinase 1], a gatekeeper of neuroinflammation (Ahmad et al., 2016), can be detected in hereditary normal tension glaucoma patients (Fingert et al., 2011). Wild-type mice could acquire glaucoma-like characteristics, such as loss of RGCs, by receiving $\mathrm{T}$ cells from $\mathrm{Sh} 3 \mathrm{pxd} 2 \mathrm{~b}^{\text {nee }}$ mice, a hereditary glaucoma model with elevated IOP (Gramlich et al., 2015). This indicates that neuroinflammation and the immune system can initiate the occurrence and progression of glaucoma.

In glaucoma, the cells involved in immunoregulation in the retina mainly include microglia, astrocytes, and Müller cells, the latter two being macroglia (Adornetto et al., 2019; Reichenbach and Bringmann, 2020). In a normal retina, these cells provide nutrition and structural support, participate in metabolism, and regulate homeostasis while coordinating with each other in the regulation of the state of neurons through phagocytosis and the secretion of inflammatory cytokines and neurotrophic factors (Vecino et al., 2016). Thus, microglia and macroglia do not function in isolation, and a delicate balance always exists between them. Glial cells work together to maintain the stability of the retinal microenvironment. However, once there is a deviation in this delicate balance, effects opposite to what is expected may occur. Therefore, understanding the interaction between glial cells plays an essential role in better glaucoma prevention and treatment.

In this review, we discuss the role and importance of microglia and macroglia in open-angle glaucoma, including glaucoma in animal models. The animal models discussed in this article are RGC loss models, most of which are high IOP models; models with RGC loss caused by other reasons are also mentioned. We analyze the contribution of the interactions between microglia and macroglia to the survival of RGCs and optic nerves with the hopes of providing additional insights into the relationship between glial cells and neurons while also providing some foundations and directions for future research. Based on the functions and characteristics of different glial cells, we propose future research directions for glial cells for better prevention and control of glaucoma.

\section{MICROGLIA IN GLAUCOMA}

Microglia, which are full-time phagocytes, play an important role in innate immune responses. Microglia are involved in neural circuits and angiogenesis in a developing retina, whereas they regulate retinal neuron activity and synaptic integrity in a mature retina (Silverman and Wong, 2018; Chen et al., 2019; Rathnasamy et al., 2019). In a healthy retina, perivascular microglia control the substances entering the retina from the circulatory system, whereas other scattered retinal microglia patrol the microenvironment to remove metabolites and cell debris and mediate synaptic remodeling (Provis et al., 1996). Microglia are distributed horizontally in the plexiform layer of the inner retina and make regular short-term contact with neuronal synapses at rest (Wake et al., 2009). They respond quickly and sensitively to pathological stimuli, such as lipopolysaccharides, complements, thrombins, inflammatory cytokines, and chemokines, migrating to an injury site within $24 \mathrm{~h}$ (Okunuki et al., 2018).

In normal human eyes, quiescent microglia, with thin ramified processes, are found around the optic nerve head $(\mathrm{ONH})$ in the walls of large blood vessels and in surrounding capillaries in glial columns and the cribriform plate. In human glaucomatous eyes, microglia are activated as clusters of large ameboids in the compressed lamina cribrosa (LC) and as formations of concentric circles surrounding blood vessels. Microglia are also redistributed to the parapapillary chorioretinal region and are present as single cells or clusters at the termination of Bruch's membrane (Neufeld, 1999). In the ONH of glaucomatous eyes, microglia express different cytokines and mediators in addition to their morphology and distribution. Research has shown that abundant transforming growth factor- $\beta 2$ (TGF- $\beta 2$ ), TNF$\alpha$, and proliferating cell nuclear antigens are present in the microglia of glaucomatous $\mathrm{ONH}$, whereas no positive signal of these factors is detected in the microglia of normal $\mathrm{ONH}$ (Yuan and Neufeld, 2001).

Microglial activation is considered one of the early alterations that occur in glaucoma. For example, in chronic hereditary glaucoma model DBA/2J mice, the aggregation, activation, and redistribution of microglia can be detected before RGC injury (Bosco et al., 2011). In DBA/2 J mice, the transcriptome of ONH microglia changes significantly in the metabolic, phagocytosis, inflammatory, and sensome pathways (Tribble et al., 2020). Among these, microglial surveillance and phagocytosis are downregulated, whereas metabolism-related transcripts are upregulated. These findings suggest that chronic ocular hypertension inhibits the homeostasis and related functions of microglia, and that $\mathrm{ONH}$ microglia increase the capacity to metabolize energy (Tribble et al., 2020). Retinal microglial reactions are regulated by many factors, such as microRNAs (miRNAs). Previous investigations have indicated that miRNAs are associated with microglial activation and polarization (Yan et al., 2015; Drewry et al., 2018; Wang et al., 2021). In a study of an acute ocular hypertension rat model, overexpression of microRNA-93-5p reduced microglial proliferation, migration, and cytokine release; these findings were accompanied by reduced loss of RGCs (Wang et al., 2021).

Despite these reported findings, the role of microglia in glaucoma remains controversial. Some researchers have suggested that microglia can aggravate RGC damage and neurodegeneration in multiple ways, including through the release of inflammatory cytokines, such as TNF- $\alpha$, interleukin $1 \beta$ (IL-1 $\beta$ ), IL-6, matrix metalloproteinases, Fas ligands, and reactive oxygen species (Hanisch and Kettenmann, 2007; Langmann, 2007). In a study of an S100B-induced glaucoma-like animal model, microglia were activated and increased in the optic nerve 
on day 14, and RGC apoptosis was observed. However, on day 21 , the microglial response was not as prominent, whereas RGC damage was still present (Grotegut et al., 2020). Therefore, the deletion of genes that affect microglial activation or other normal functions can contribute to the survival of RGCs or the integrity of the optic nerve. Microglia express high levels of complement peptide C3a receptor 1 (C3ar1), which has been identified as a damaging neuroinflammatory factor. Deficiency in C3ar1 lowers the risk of retinal degeneration independent of IOP in DBA/2J mice (Harder et al., 2020). The neutralization or genetic deletion of TNF- $\alpha$ significantly reduces RGC loss in a glaucoma mouse model with angle closure induced via argon laser irradiation (Nakazawa et al., 2006). Deletion of the CD11b/CD18 gene, which mediates the recruitment and activation of leukocytes, inhibits the loss of RGCs and combats neurotoxicity (Nakazawa et al., 2006). Meanwhile, a deficiency in $\mathrm{C}-\mathrm{X} 3-\mathrm{C}$ motif chemokine receptor 1 (CX3CR1), which is expressed by neurons, can inhibit microglial reactivity, aggravate neurotoxicity, and induce extensive damage to RGCs in an experimental mouse glaucoma model with transient elevation of IOP (Wang K. et al., 2014). Some drug interventions can also exert beneficial effects on RGC survival by inhibiting microglial reactivity. Minocycline, a drug that reduces microglial activation, improves the integrity of RGC axons and the function of the optic nerve in DBA/2J mice (Bosco et al., 2008). Intravitreal injection of macrophage inhibitory factor in a rat optic nerve axotomy model (Thanos et al., 1993) and high-dose irradiation of the head of a DBA/2J mouse (Bosco et al., 2012) inhibit the degradation of neurons and promote the development of axons. Furthermore, the proliferation and activation of microglia has shown a significant correlation with the severity of optic neuropathy in DBA/2J mice (Bosco et al., 2015).

However, some studies have suggested that microglia can protect RGCs from damage. Microglia help to prevent secondary retinal damage by phagocytosing damaged or dead RGCs in the later stages of optic nerve damage (Sierra et al., 2013). It is well known that DNA can act as danger-associated molecular patterns, inducing tissue toxicity and organ damage in a mouse glaucoma model with transient elevation of IOP (Supinski et al., 2020). As major phagocytes, microglia can prevent DNA release from dying cells through phagocytosis (Egensperger et al., 1996). Once activated, microglia can clear multiple apoptotic RGCs for at least 14 days after reaxotomy of the RGCs (Schuetz and Thanos, 2004); however, inappropriate phagocytosis by microglia can accelerate the loss of RGCs (Brown and Neher, 2014). Therefore, regulation of phagocytosis enables microglia to function better as guardians during retinal injury. In addition, microglia produce neurotrophic factors (Harada et al., 2002), remove excess glutamate (Basilico et al., 2019), and exert antioxidant effects through antioxidant enzymes in the cells (Vilhardt et al., 2017) when the retina is damaged.

In summary, microglia exert neuroprotective or neurotoxic effects in glaucomatous eyes, depending on the secreted factors. Microglia are activated in the early stages of glaucoma and rapidly migrate to an injury site. They stabilize the retinal microenvironment through phagocytosis and secretion of antiinflammatory cytokines, thus protecting the optic nerve and
RGCs. In the late stage of glaucoma, however, overactivated microglia produce pro-inflammatory cytokines, complement, and other toxic factors that directly affect RGC apoptosis. There is no doubt that microglia are an important target for the prevention of glaucoma, and due to their complexity, more accurate and precise intervention and regulation involving them will be a challenge in future research.

\section{MACROGLIA IN GLAUCOMA}

Macroglia, which mainly include astrocytes and Müller cells, possess similar transcription profiles and functions (Xue et al., 2011). Their cell bodies surround the axons of neurons and form glial sheaths around neurons that protect and buffer them. Müller cells span the entire retinal layer, whereas astrocytes are confined to the innermost layer of the retina. The difference between their distribution indicates that their monitoring and reacting areas are different; Müller cells are more global, whereas astrocytes are local. Their close contact with neurons also allows macroglia to sensitively perceive changes in the microenvironment, such as hypoxia, and respond in a timely manner. Macroglia transport most nutrients, wastes, ions, water, and other molecules between blood vessels and neurons (Reichenbach and Bringmann, 2020). In addition, they contribute to various glial homeostatic functions, such as the regulation of extracellular $\mathrm{pH}$ and potassium balance (Agte et al., 2011).

When the retina is stimulated or injured, macroglia can be activated and can express glial fibrillary acidic protein (GFAP) and other extracellular matrix signals through enlarged cell bodies and thickened foot processes (Varela and Hernandez, 1997; Tezel et al., 2003; Lukowski et al., 2019), a process known as retinal gliosis. In a human glaucomatous retina, astrocytes and Müller cells show a hypertrophic morphology with increased immunostaining of GFAP, which suggests that retinal gliosis is exhibited in glaucoma (Tezel et al., 2003).

\section{Astrocytes in Glaucoma}

As the main component of glial cells in the central nervous system, astrocytes play a variety of essential roles, including the regulation of ion balance, metabolic supply and structural support, neurotransmitter transmission, and synaptic plasticity (Halassa et al., 2007). Astrocytes not only play the role of maintaining retinal homeostasis but also directly act on the pathophysiology of RGCs and the optic nerve through a variety of factors.

In clinical practice, optic disk cupping can be easily discernible in glaucomatous eyes. ONH cupping can be induced by structural changes in the LC, which is enriched with collagens (Elkington et al., 1990). Optic nerve axons in the LC are unmyelinated and surrounded by astrocytes (Shinozaki and Koizumi, 2021). Activated astrocytes have been found in the human glaucomatous retina (Minckler and Spaeth, 1981; Tezel et al., 2003). Recently, intravitreal injection of $\mathrm{S} 100 \mathrm{~B}$, which is mainly expressed by astrocytes, was found to cause glaucoma-like neuronal degeneration in the retina and optic nerve (Grotegut et al., 2020). 
In glaucoma, astrocytes change from a resting state to an activated state in a process known as astrogliosis and act as early responders to induce inflammatory responses. Astrocytes have been shown to mediate the activation of multiple inflammatory pathways, including TNF- $\alpha$ signaling, NF-кB activation, autophagy, and inflammasome-associated regulators in high IOP rats after administration of hypertonic saline injections into their episcleral veins (Tezel et al., 2012). In patients with glaucoma and glaucoma animal models, astrocytes at the ONH can be observed to upregulate the expression of tenascinC (Pena et al., 1999; Howell et al., 2011; Johnson et al., 2011), playing a pro-inflammatory role through the Toll-like receptor (TLR) 4 signaling pathway (Midwood et al., 2009). Astrocytes also induce the adhesion and migration of immune cells by secreting cell adhesion proteins (Johnson et al., 2011; Tanigami et al., 2012). They participate in the remodeling of the extracellular matrix of the LC, which is related to a variety of molecular pathways, including those for TGF- $\beta$, endothelins, bone morphogenetic proteins, and gremlin (Schneider and Fuchshofer, 2016).

In response to an increase in IOP, astrocytes expand and redistribute metabolites, donating resources from unstressed projections to stressed projections in DBA/2J mice (Cooper et al., 2020). Furthermore, once IOP is increased, activated astrocytes accompanied by upregulated cytoskeletal protein concentrate and tightly surround the ONH; however, long-term high IOP causes the astrocytes around the optic nerve to decrease or even disappear after magnetic microsphere injection (Dai et al., 2012). In the early stage of glaucoma progression in DBA/2J mice models, astrocytes become more parallel with migration to the edge of the nerve; however, the astrocytes' parallelism diminishes as axons degenerate and glial coverage increases (Cooper et al., 2018). Moreover, some studies have shown that ONH astrocytes play a role in the engulfment of optic axons and promotion of axon degradation, which is one of the possible mechanisms for the sectorial nature of RGC loss in glaucoma (Nguyen et al., 2011; Mills et al., 2015).

Astrogliosis is beneficial to the retina, and its morphological remodeling is reversible in the early stages of the disease. However, in the late stage, it can cause excessive scar hyperplasia and degrade optic axons, which are detrimental to the repair of the body (Sun et al., 2013).

\section{Müller Cells in Glaucoma}

Müller cells are the most abundant glial cells in the retina and are closely related to RGCs in anatomy and structure. They play a crucial role in neuronal development, survival, and related information processing (Bringmann et al., 2006). The cell body of a Müller cell is located in the inner nuclear layer, and its processes extend to the inner and outer layers of the retina. Müller cells tightly contact synapses and blood vessels in the inner and outer plexiform layers of the retina. Müller cells are rich in different ion channels, ligand receptors, transmembrane transport molecules, and enzymes (Bringmann et al., 2006). Their structural richness determines the diversity of their functions. Regarding physiological conditions, Müller cells are involved in retinal glucose metabolism, regulation of retinal blood flow and neurotransmitter transmission, and regulation of the balance of ions, water, and amino acids (Bringmann et al., 2006). Regarding pathological conditions, Müller cells regulate immunity and phagocytic cells or foreign substances. In particular, Müller cells undergo gliosis to cope with various types of retinopathy (Harada et al., 2002).

Müller cell reactions are detectable in the human glaucomatous retina through immunohistochemistry (Tezel et al., 2003). Müller cells regulate intracellular and extracellular glutamate levels through the expression of glutamate/aspartate transporter (GLAST) (Rauen, 2000). A study of GLAST knockout mice indicated that they showed spontaneous RGC loss and glaucomatous optic nerve degeneration without elevated IOP (Harada et al., 2007).

Müller cell gliosis mainly plays a protective role in the early stages of glaucoma. Investigation using scanning force microscopy has shown that Müller cells have very soft and flexible bodies with strong plasticity and powerful compliance (Lu et al., 2006). Furthermore, their hypertrophic cell bodies and increased intermediate filaments, such as GFAP, enhance resistance to mechanical forces, including elevated IOP (Lu et al., 2006). The expression of neurotrophins and their receptors can be detected in Müller cells after retinal ischemia-reperfusion (Vecino et al., 1998). In addition, Müller cells secrete neurotrophic factors, such as ciliary neurotrophic factor (CNTF), and the antioxidants glutathione (Pease et al., 2009) and ghrelin (Zhu et al., 2017), protecting RGCs from damage in glaucoma.

In the later stages of glaucoma, however, over-gliosis of Müller cells is dysfunctional, leading to disturbances in retinal homeostasis and neuronal death. Müller cells show excitotoxic damage to RGCs because their degradation of glutamic acid is weakened during hypoxia (Evangelho et al., 2019). Owing to the damage of the Müller cell membrane under high pressure, $\mathrm{K}^{+}$siphoning is impaired, inducing cation dyshomeostasis in the microbead occlusion model (Fischer et al., 2019). They are not sufficient to rebalance microenvironmental changes and even activate neuronal pro-apoptotic pathways. Müller cell activation also aggravates the damage of RGCs through NF- $\kappa \mathrm{B}$-dependent TNF- $\alpha$ production (Lebrun-Julien et al., 2009), indicating that Müller cells indirectly contribute to neuroinflammation.

\section{INTERACTION BETWEEN MACROGLIA AND MICROGLIA IN GLAUCOMA}

Although the origins and main functions of microglia and macroglia are different, they coordinate with each other to complete the nutrition, support, and protection of neurons (Figure 1). In a mature retina, macroglia, microglia, and pericytes inhibit the proliferation of endothelial cells and maintain the stability of blood vessels and the inner blood-retinal barrier (Gardner et al., 1997; West et al., 2005; Klaassen et al., 2013). In a healthy retina, the state of microglial activation depends directly on extracellular adenosine triphosphate (ATP). The main source of ATP is Müller cells, which means that Müller cells can indirectly serve as an energy source for microglia (Stout et al., 2002; Pankratov et al., 2006; Uckermann et al., 2006; Li et al., 2012). 


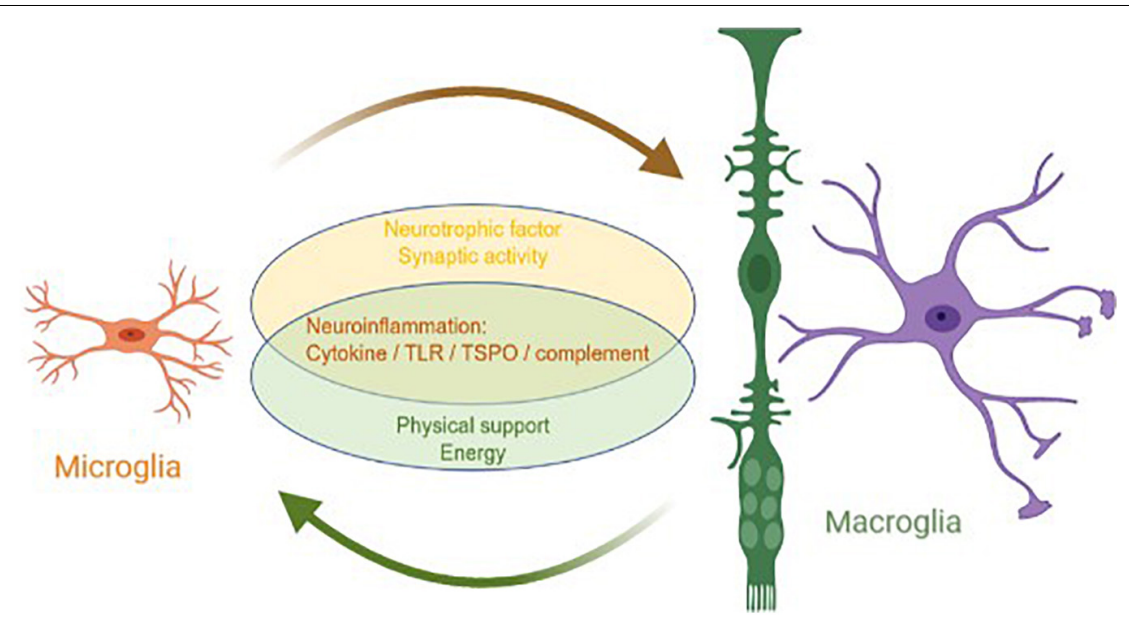

FIGURE 1 | Interaction between macroglia and microglia in glaucoma. Microglia and macroglia are involved in the progression of neuroinflammation in glaucoma, including the inflammatory cytokine, TLR, TSPO, and complement pathways. In addition, microglia prompt macroglia to produce neurotrophic factors and help regulate synaptic activity, whereas macroglia provide microglia with the physical scaffold support and energy required for activities.

After the retina is mechanically damaged or becomes degenerative, the interaction between microglia and macroglia begins immediately, and then the timing and extent of microglial activation is closely regulated (Wang M. et al., 2014). There are some similarities in the changes that occur in microglia and macroglia in glaucoma. In a study of IOP-independent experimental autoimmune glaucoma (EAG) mice models, the mice were immunized with an optic nerve antigen (ONA). Compared with the control group, the EAG mice displayed fewer Brna+ RGCs and SMI-31+ optic nerve neurofilaments, with a significantly higher number of retinal Ibal + microglia and GFAP + astrocytes (Wiemann et al., 2020).

In a resting state, microglia mainly exhibit horizontal movement. However, with retinal injury or other stress, they begin vertical transretinal movement, which is closer to the behavior of Müller cells. Injection of lipopolysaccharides into the vitreous cavity of mice has shown that the horizontal branches of the microglia are in close contact with the cell bodies of Müller cells, suggesting that microglia may use Müller cells as scaffolds for transretinal migration (Sánchez-López et al., 2004; Wang et al., 2011). Müller cells co-cultured with activated microglia show more elongated spindles and multipolar shapes than their flat, thick lamellipodia when cultured alone (Wang and Wong, 2014). In addition, under stress, gliosis in retinal glia can be triggered by microglial activation through increased cytokine levels (Dharmarajan et al., 2017). Moreover, IL-6 (Fischer et al., 2014; Zhao et al., 2014) and interferon$\gamma($ IFN- $\gamma)$ (Cotinet et al., 1997) cause morphological changes and promote the production of inflammatory factors in Müller cells. In zebrafish, the pharmacological ablation of microglia results in a lack of reactivity in Müller cells with less GFAP expression; however, it does not affect the migration of Müller cells (Conedera et al., 2019). It has also been found that progesterone can reduce Müller cell gliosis by inhibiting the expression of IFN- $\gamma$ from microglia in a retinitis pigmentosa mouse model (Roche et al., 2018). Activated microglia also regulate Müller cells to produce and release more trophic factors, such as basic fibroblast growth factor (bFGF) (Harada et al., 2002), glial-derived neurotrophic factor (GDNF), and leukemia inhibitory factor (LIF), thereby amplifying the protective effects of Müller cells. This response is independent of the expression of the typical glial markers of Müller cells (Pease et al., 2009). Activated microglia also participate in the maintenance of the synaptic activity microenvironment of macroglia, and they jointly regulate the content of ions and neurotransmitters (Kimelberg, 2010; Chong and Martin, 2015; Ma et al., 2019). Glial cells also contribute to the activation of the complement pathway in glaucoma (Pauly et al., 2019). The level of complements in Müller cells and microglia increases with transient retinal ischemia (Pauly et al., 2019). In addition, astrocytes activate C3 convertase, thereby amplifying complement signals and alerting microglia to respond to RGC damage (Howell et al., 2011; Astafurov et al., 2014; Williams et al., 2017).

Traditionally, microglia and astrocytes are considered to have two different phenotypes. Microglia are classified into "M1" and "M2" phenotypes. Simply put, M1 is an inflammationpromoting phenotype, whereas M2 is an anti-inflammatory phenotype (Varnum and Ikezu, 2012; Tang and Le, 2016). In animal models of laser-induced glaucoma, most microglial cells exhibit the M1 phenotype (Rojas et al., 2014). Similarly, astrocytes are categorized into "A1" and "A2" phenotypes. The A1 phenotype aggravates neuroinflammation, whereas A2 is neuroprotective (Liddelow et al., 2017). M1 and M2 microglia can stimulate reactive astrocyte changes by secreting different cytokines (Wei et al., 2019). M1 microglia, which secrete pro-inflammatory cytokines, can promote astrocytes to switch to the A1 phenotype, thereby increasing neurotoxicity and inducing neuronal death (Liddelow et al., 2017). Recently, singlecell RNA sequencing, which provides an unbiased analysis of cells, has been used to uncover more information about the heterogeneity of astrocytes and microglia. In studies of experimental autoimmune encephalomyelitis (EAE), astrocytes 
were identified as several subpopulations, and pro-inflammatory and neurotoxic subpopulations were the most expanded (Linnerbauer et al., 2020; Wheeler et al., 2020). Microglia show a variable spatial and temporal distribution during development and can gain a discrete context- and time-dependent diseasespecific signature in the central nervous system pathology of mice. Corresponding clusters of microglia have also been identified in the brains of patients with multiple sclerosis (Masuda et al., 2019).

In addition to the aforementioned unidirectional coordination between glial cells, microglia and Müller cells are likely to have bidirectional feedback signals in response to injury or other stress. For example, activated microglia induce Müller cells to secrete more adhesion molecules and chemokines so that more microglia can adhere to the Müller cells and express higher chemotaxis, which also promotes microglial migration and recruits other immune cells to the injured areas (Evangelho et al., 2019). The upregulation of TLR signals in both microglia and astrocytes has been found in the retina of patients with glaucoma (Luo et al., 2010), and together they may mediate the initial immunity initiated by glaucoma stimulation signals. Both phagocytic microglia (Yuan and Neufeld, 2001) and reactive astrocytes (Zhang et al., 2004) express metalloproteinases, which increase during optic neuropathy, altering the amount of extracellular matrix to mediate retinal degeneration. Translocator protein (TSPO) and its ligand XBD173 (Mages et al., 2019) may be key mediators of the inflammatory response between microglia and macroglia (Scholz et al., 2015; Cooper et al., 2020). As initiators and amplifiers of retinal inflammation, activated microglia and Müller cells (Scholz et al., 2015) can mutually and reciprocally promote each other to produce more inflammatory cytokines, thereby creating a positive feedback loop and exacerbating the inflammatory response (Wang et al., 2011).

Generally, microglia are the main executors of neuroinflammation in a glaucomatous retina, whereas macroglia are supporters. Both microglia and macroglia undergo changes in morphology and physical location when the retina is under stress. As the only antigen-presenting cells of the retina, microglia are activated when an injury occurs and quickly migrate to the injury site. As the main supporting cells of the retina, macroglia also become mobilized and move parallel to the edge of the nerve, redistributing resources in response to the injury. These two types of glial cells coordinate to mediate neuroinflammation, including neuroinflammation from the cytokine, TLR, TSPO, and complement signaling pathways. Microglia are mainly involved in neuroinflammation, whereas macroglia regulate neuroinflammation in various ways. Furthermore, microglia prompt macroglia to produce neurotrophic factors and help regulate synaptic activity, whereas macroglia provide microglia with the physical scaffold support and ATP required for activities.

\section{GLIA-TARGETED THERAPEUTIC APPROACHES}

Overactivation of microglia is considered detrimental to the survival of RGCs. Therefore, protecting RGC by inhibiting the activity of microglia is an effective treatment for glaucoma. Some drug interventions exert beneficial effects on RGC survival by blocking microglial-relevant pathways. Microglia inhibitors have been shown to effectively protect RGCs in animal models (Drewry et al., 2018; Grotegut et al., 2020; Supinski et al., 2020). At present, microglia inhibitors have not been applied to the treatment of glaucoma. However, they have been used for the management of other diseases caused by retinal inflammation, an important aggravating factor for glaucoma. Treatment with the microglial inhibitory agent minocycline is associated with improved visual function, central macular edema, and vascular leakage, targeting the inflammatory etiology of diabetic macular edema in humans (Cukras et al., 2012).

In recent studies, macroglia have shown the potential for reprogramming and re-differentiation, which brings new hope for the treatment of retinal diseases. Glial cells and neurons are derived from the same pluripotent stem cells, and the influence of extracellular signals on them leads to different outcomes (Janowska et al., 2019). In theory, by regulating the extracellular signals of glial cells, their fate can be altered, and they can re-differentiate into glial cells or neurons. Targeting glia for neurorestorative therapy is a safe, effective, and efficient strategy. In the early stage of glaucoma, astrocytes and Müller cells limit the spread of inflammation and the progression of glaucoma. In the late stage of the disease, the overactive glial cells would form glial scars, which aggravate the progression of glaucoma (Reichenbach and Bringmann, 2020). Judging and selecting the appropriate intervention period may be the direction for the exploration of the astrocyte-based treatment in the future. In zebrafish, Müller cells enter a reprogramming state relatively easily, whereas in mammals, they enter a reactive state after injury; however, they stagnate before becoming progenitor cells (Hoang et al., 2020). How to regulate the entrance of mammalian Müller cells into the reprogramming state will be a future research direction.

\section{CONCLUSION}

Many studies of patients with glaucoma and glaucoma animal models have shown that optic nerve damage or high IOP can cause the production of inflammatory cytokines and chemokines, as well as the activation of ocular immune cells, such as microglia and macroglia. A delicate balance between glial cells is critical for the development and maturation of the retina under normal conditions and during the pathological process of disease conditions. The neuroinflammation mediated by these cells is one of the key factors in the onset and progression of glaucoma. There is no doubt that IOP reduction is an effective prevention and treatment measure for patients with high-tension glaucoma. However, the roles of controlled neuroinflammation and promotion of neurotrophy in the treatment of glaucoma are also worth considering. However, microglia and macroglia do not function in isolation in glaucoma. Furthermore, an understanding of the interactions between glial cells is necessary for the development of new interventions to reduce neuroinflammation and prevent 
glaucoma. More research is needed to better understand the complex molecular spatiotemporal regulatory network in glaucoma. Further elucidation of the mechanism and response of glial cells in glaucoma will have a significant impact on the development of new cell-based therapies for retinal diseases.

\section{AUTHOR CONTRIBUTIONS}

All authors read and approved the final manuscript.

\section{REFERENCES}

Adornetto, A., Russo, R., and Parisi, V. (2019). Neuroinflammation as a target for glaucoma therapy. Neural Regen. Res. 14, 391-394. doi: 10.4103/1673-5374. 245465

Agte, S., Junek, S., Matthias, S., Ulbricht, E., Erdmann, I., Wurm, A., et al. (2011). Müller glial cell-provided cellular light guidance through the vital guinea-pig retina. Biophys. J. 101, 2611-2619. doi: 10.1016/j.bpj.2011.09.062

Ahmad, L., Zhang, S. Y., Casanova, J. L., and Sancho-Shimizu, V. (2016). Human TBK1: a gatekeeper of neuroinflammation. Trends Mol. Med. 22, 511-527. doi: 10.1016/j.molmed.2016.04.006

Astafurov, K., Elhawy, E., Ren, L., Dong, C. Q., Igboin, C., Hyman, L., et al. (2014). Oral microbiome link to neurodegeneration in glaucoma. PLoS One 9:e104416. doi: 10.1371/journal.pone. 0104416

Basilico, B., Pagani, F., Grimaldi, A., Cortese, B., Di Angelantonio, S., Weinhard, L., et al. (2019). Microglia shape presynaptic properties at developing glutamatergic synapses. Glia 67, 53-67. doi: 10.1002/glia.23508

Bosco, A., Crish, S. D., Steele, M. R., Romero, C. O., Inman, D. M., Horner, P. J., et al. (2012). Early reduction of microglia activation by irradiation in a model of chronic glaucoma. PLoS One 7:e43602. doi: 10.1371/journal.pone.004 3602

Bosco, A., Inman, D. M., Steele, M. R., Wu, G., Soto, I., Marsh-Armstrong, N., et al. (2008). Reduced retina microglial activation and improved optic nerve integrity with minocycline treatment in the DBA/2J mouse model of glaucoma. Invest. Ophthalmol. Vis. Sci. 49, 1437-1446. doi: 10.1167/iovs.07-1337

Bosco, A., Romero, C. O., Breen, K. T., Chagovetz, A. A., Steele, M. R., Ambati, B. K., et al. (2015). Neurodegeneration severity can be predicted from early microglia alterations monitored in vivo in a mouse model of chronic glaucoma. Dis. Model. Mech. 8, 443-455. doi: 10.1242/dmm.018788

Bosco, A., Steele, M. R., and Vetter, M. L. (2011). Early microglia activation in a mouse model of chronic glaucoma. J. Comp. Neurol. 519, 599-620. doi: $10.1002 /$ cne.22516

Bringmann, A., Pannicke, T., Grosche, J., Francke, M., Wiedemann, P., Skatchkov, S. N., et al. (2006). Müller cells in the healthy and diseased retina. Prog. Retin. Eye Res. 25, 397-424.

Brown, G. C., and Neher, J. J. (2014). Microglial phagocytosis of live neurons. Nat. Rev. Neurosci. 15, 209-216. doi: 10.1038/nrn3710

Calkins, D. J. (2021). Adaptive responses to neurodegenerative stress in glaucoma. Prog. Retin. Eye Res. 2021:100953. doi: 10.1016/j.preteyeres.2021.100953

Chen, M., Luo, C., Zhao, J., Devarajan, G., and Xu, H. (2019). Immune regulation in the aging retina. Prog. Retin. Eye Res. 69, 159-172. doi: 10.1016/j.preteyeres. 2018.10.003

Chong, R. S., and Martin, K. R. (2015). Glial cell interactions and glaucoma. Curr. Opin. Ophthalmol. 26, 73-77. doi: 10.1097/icu.0000000000000125

Conedera, F. M., Pousa, A. M. Q., Mercader, N., Tschopp, M., and Enzmann, V. (2019). Retinal microglia signaling affects Müller cell behavior in the zebrafish following laser injury induction. Glia 67, 1150-1166. doi: 10.1002/glia.23601

Conlon, R., Saheb, H., and Ahmed, I. I. K. (2017). Glaucoma treatment trends: a review. J. Can. D'ophtalmol. 52, 114-124. doi: 10.1016/j.jcjo.2016.07.013

Cooper, M. L., Collyer, J. W., and Calkins, D. J. (2018). Astrocyte rem odeling without gliosis precedes optic nerve Axonopathy. Acta Neuropathol. Commun. 6:38.

Cooper, M. L., Pasini, S., Lambert, W. S., D’Alessandro, K. B., Yao, V., Risner, M. L., et al. (2020). Redistribution of metabolic resources through astrocyte

\section{FUNDING}

This study was supported by grants from the National Key Research and Development Program of China (SQ2018 YFA090045-01, 2017YFA0105301, and 2019ZX09301113), the National Natural Science Foundation of China (81771739 and 81730026), the Science and Technology Commission of Shanghai Municipality (17411953000 and 19495800700), and the Program for Professor of Special Appointments (Eastern Scholar) at Shanghai Institutions of Higher Learning.

networks mitigates neurodegenerative stress. Proc. Natl. Acad. Sci. U.S.A. 117, 18810-18821. doi: 10.1073/pnas.2009425117

Cotinet, A., Goureau, O., Hicks, D., Thillaye-Goldenberg, B., and de Kozak, Y. (1997). Tumor necrosis factor and nitric oxide production by retinal Müller glial cells from rats exhibiting inherited retinal dystrophy. Glia 20, 59-69. doi: 10.1002/(sici)1098-1136(199705)20:1<59::aid-glia6>3.0.co;2-0

Cukras, C. A., Petrou, P., Chew, E. Y., Meyerle, C. B., and Wong, W. T. (2012). Oral minocycline for the treatment of diabetic macular edema (DME): results of a phase I/II clinical study. Invest. Ophthalmol. Vis. Sci. 53, 3865-3874. doi: 10.1167/iovs.11-9413

Dai, C., Khaw, P. T., Yin, Z. Q., Li, D., Raisman, G., and Li, Y. (2012). Structural basis of glaucoma: the fortified astrocytes of the optic nerve head are the target of raised intraocular pressure. Glia 60, 13-28. doi: 10.1002/glia.21242

Dharmarajan, S., Fisk, D. L., Sorenson, C. M., Sheibani, N., and Belecky-Adams, T. L. (2017). Microglia activation is essential for BMP7-mediated retinal reactive gliosis. J. Neuroinflamm. 14:76.

Drewry, M. D., Challa, P., Kuchtey, J. G., Navarro, I., Helwa, I., Hu, Y., et al. (2018). Differentially expressed microRNAs in the aqueous humor of patients with exfoliation glaucoma or primary open-angle glaucoma. Hum. Mol. Genet. 27, 1263-1275. doi: 10.1093/hmg/ddy040

Egensperger, R., Maslim, J., Bisti, S., Holländer, H., and Stone, J. (1996). Fate of DNA from retinal cells dying during development: uptake by microglia and macroglia (Müller cells). Brain Res. Dev. Brain Res. 97, 1-8. doi: 10.1016/s01653806(96)00119-8

Elkington, A. R., Inman, C. B., Steart, P. V., and Weller, R. O. (1990). The structure of the lamina cribrosa of the human eye: an immunocytochemical and electron microscopical study. Eye 4(Pt 1), 42-57. doi: 10.1038/eye.1990.5

Evangelho, K., Mogilevskaya, M., Losada-Barragan, M., and Vargas-Sanchez, J. K. (2019). Pathophysiology of primary open-angle glaucoma from a neuroinflammatory and neurotoxicity perspective: a review of the literature. Int. Ophthalmol. 39, 259-271. doi: 10.1007/s10792-017-0795-9

Fingert, J. H., Robin, A. L., Stone, J. L., Roos, B. R., Davis, L. K., Scheetz, T. E., et al. (2011). Copy number variations on chromosome 12q14 in patients with normal tension glaucoma. Hum. Mol. Genet. 20, 2482-2494. doi: 10.1093/hmg/ddr123

Fischer, A. J., Zelinka, C., Gallina, D., Scott, M. A., and Todd, L. (2014). Reactive microglia and macrophage facilitate the formation of Müller glia-derived retinal progenitors. Glia 62, 1608-1628. doi: 10.1002/glia.22703

Fischer, R. A., Roux, A. L., Wareham, L. K., and Sappington, R. M. (2019). Pressuredependent modulation of inward-rectifying $\mathrm{K}(+)$ channels: implications for cation homeostasis and $\mathrm{K}(+)$ dynamics in glaucoma. Am. J. Physiol. Cell Physiol. 317, C375-C389.

Gardner, T. W., Lieth, E., Khin, S. A., Barber, A. J., Bonsall, D. J., Lesher, T., et al. (1997). Astrocytes increase barrier properties and ZO-1 expression in retinal vascular endothelial cells. Invest. Ophthalmol. Vis. Sci. 38, 2423-2427.

Gordon, M. O., and Kass, M. A. (2018). What we have learned from the ocular hypertension treatment study. Am. J. Ophthalmol. 189, 24-27.

Gramlich, O. W., Ding, Q. J., Zhu, W., Cook, A., Anderson, M. G., and Kuehn, M. H. (2015). Adoptive transfer of immune cells from glaucomatous mice provokes retinal ganglion cell loss in recipients. Acta Neuropathol. Commun. 3:56.

Grotegut, P., Kuehn, S., Meißner, W., Dick, H. B., and Joachim, S. C. (2020). Intravitreal S100B injection triggers a time-dependent microglia response in a pro-inflammatory manner in retina and optic nerve. Mol. Neurobiol. 57, 1186-1202. doi: 10.1007/s12035-019-01786-4 
Halassa, M. M., Fellin, T., and Haydon, P. G. (2007). The tripartite synapse: roles for gliotransmission in health and disease. Trends Mol. Med. 13, 54-63. doi: 10.1016/j.molmed.2006.12.005

Hanisch, U. K., and Kettenmann, H. (2007). Microglia: active sensor and versatile effector cells in the normal and pathologic brain. Nat. Neurosci. 10, 1387-1394. doi: 10.1038/nn 1997

Harada, T., Harada, C., Kohsaka, S., Wada, E., Yoshida, K., Ohno, S., et al. (2002). Microglia-Müller glia cell interactions control neurotrophic factor production during light-induced retinal degeneration. J. Neurosci. 22, 9228-9236. doi: 10.1523/jneurosci.22-21-09228.2002

Harada, T., Harada, C., Nakamura, K., Quah, H. M., Okumura, A., Namekata, K., et al. (2007). The potential role of glutamate transporters in the pathogenesis of normal tension glaucoma. J. Clin. Invest. 117, 1763-1770. doi: 10.1172/jci30178

Harder, J. M., Williams, P. A., Braine, C. E., Yang, H. S., Thomas, J. M., Foxworth, N. E., et al. (2020). Complement peptide C3a receptor 1 promotes optic nerve degeneration in DBA/2J mice. J. Neuroinflamm. 17:336.

Hoang, T., Wang, J., Boyd, P., Wang, F., Santiago, C., Jia, L., et al. (2020). Gene regulatory networks controlling vertebrate retinal regeneration. Science 370:eabb8598. doi: 10.1126/science.abb8598

Howell, G. R., Macalinao, D. G., Sousa, G. L., Walden, M., Soto, I., Kneeland, S. C., et al. (2011). Molecular clustering identifies complement and endothelin induction as early events in a mouse model of glaucoma. J. Clin. Invest. 121, 1429-1444. doi: 10.1172/jci44646

Janowska, J., Gargas, J., Ziemka-Nalecz, M., Zalewska, T., Buzanska, L., Sypecka, J., et al. (2019). Directed glial differentiation and transdifferentiation for neural tissue regeneration. Exper. Neurol. 319:112813. doi: 10.1016/j.expneurol.2018. 08.010

Johnson, E. C., Doser, T. A., Cepurna, W., Dyck, J. A., Jia, L., Guo, Y., et al. (2011). Cell proliferation and interleukin-6-type cytokine signaling are implicated by gene expression responses in early optic nerve head injury in rat glaucoma. Invest. Ophthalmol. Vis. Sci. 52, 504-518. doi: 10.1167/iovs.10-5317

Kimelberg, H. K. (2010). Functions of mature mammalian astrocytes: a current view. Neuroscientist 16, 79-106. doi: 10.1177/1073858409342593

Klaassen, I., Van Noorden, C. J. F., and Schlingemann, R. O. (2013). Molecular basis of the inner blood-retinal barrier and its breakdown in diabetic macular edema and other pathological conditions. Prog. Retin. Eye Res. 34, 19-48. doi: 10.1016/j.preteyeres.2013.02.001

Langmann, T. (2007). Microglia activation in retinal degeneration. J. Leukoc. Biol. 81, 1345-1351. doi: 10.1189/jlb.0207114

Lebrun-Julien, F., Duplan, L., Pernet, V., Osswald, I., Sapieha, P., Bourgeois, P., et al. (2009). Excitotoxic death of retinal neurons in vivo occurs via a non-cellautonomous mechanism. J. Neurosci. 29, 5536-5545. doi: 10.1523/jneurosci. 0831-09.2009

Li, Y., Du, X. F., Liu, C. S., Wen, Z. L., and Du, J. L. (2012). Reciprocal regulation between resting microglial dynamics and neuronal activity in vivo. Dev. Cell 23, 1189-1202. doi: 10.1016/j.devcel.2012.10.027

Liddelow, S. A., Guttenplan, K. A., Clarke, L. E., Bennett, F. C., Bohlen, C. J., Schirmer, L., et al. (2017). Neurotoxic reactive astrocytes are induced by activated microglia. Nature 541, 481-487.

Linnerbauer, M., Wheeler, M. A., and Quintana, F. J. (2020). Astrocyte crosstalk in CNS inflammation. Neuron 108, 608-622. doi: 10.1016/j.neuron.2020.08.012

Lu, Y. B., Franze, K., Seifert, G., Steinhäuser, C., Kirchhoff, F., Wolburg, H., et al. (2006). Viscoelastic properties of individual glial cells and neurons in the CNS. Proc. Natl. Acad. Sci. U.S.A. 103, 17759-17764. doi: 10.1073/pnas.0606150103

Lukowski, S. W., Lo, C. Y., Sharov, A. A., Nguyen, Q., Fang, L., Hung, S. S., et al. (2019). A single-cell transcriptome atlas of the adult human retina. EMBO J. 38:e100811.

Luo, C., Yang, X., Kain, A. D., Powell, D. W., Kuehn, M. H., and Tezel, G. (2010). Glaucomatous tissue stress and the regulation of immune response through glial Toll-like receptor signaling. Invest. Ophthalmol. Vis. Sci. 51, 5697-5707. doi: 10.1167/iovs.10-5407

Ma, W., Silverman, S. M., Zhao, L., Villasmil, R., Campos, M. M., Amaral, J., et al. (2019). Absence of TGF $\beta$ signaling in retinal microglia induces retinal degeneration and exacerbates choroidal neovascularization. eLife 8:e42049.

Mages, K., Grassmann, F., Jägle, H., Rupprecht, R., Weber, B. H. F., Hauck, S. M., et al. (2019). The agonistic TSPO ligand XBD173 attenuates the glial response thereby protecting inner retinal neurons in a murine model of retinal ischemia. J. Neuroinflamm. 16:43.
Masuda, T., Sankowski, R., Staszewski, O., Böttcher, C., Amann, L., Scheiwe, C., et al. (2019). Spatial and temporal heterogeneity of mouse and human microglia at single-cell resolution. Nature 566, 388-392. doi: 10.1038/s41586-019-0924-x

Midwood, K., Sacre, S., Piccinini, A. M., Inglis, J., Trebaul, A., Chan, E., et al. (2009). Tenascin-C is an endogenous activator of Toll-like receptor 4 that is essential for maintaining inflammation in arthritic joint disease. Nat. Med. 15, 774-780. doi: $10.1038 / \mathrm{nm} .1987$

Mills, E. A., Davis, C. H., Bushong, E. A., Boassa, D., Kim, K. Y., Ellisman, M. H., et al. (2015). Astrocytes phagocytose focal dystrophies from shortening myelin segments in the optic nerve of Xenopus laevis at metamorphosis. Proc. Natl. Acad. Sci. U.S.A. 112, 10509-10514. doi: 10.1073/pnas.1506486112

Minckler, D. S., and Spaeth, G. L. (1981). Optic nerve damage in glaucoma. Surv. Ophthalmol. 26, 128-148.

Nakazawa, T., Nakazawa, C., Matsubara, A., Nakazawa, C. Y., de Paula, T. A. O., Zamper, R. P. C., et al. (2006). Tumor necrosis factor-alpha mediates oligodendrocyte death and delayed retinal ganglion cell loss in a mouse model of glaucoma. J. Neurosci. 26, 12633-12641. doi: 10.1523/jneurosci.2801-06. 2006

Neufeld, A. H. (1999). Microglia in the optic nerve head and the region of parapapillary chorioretinal atrophy in glaucoma. Arch. Ophthalmol. 117, 10501056. doi: 10.1001/archopht.117.8.1050

Nguyen, J. V., Soto, I., Kim, K. Y., Bushong, E. A., Oglesby, E., Valiente-Soriano, F. J., et al. (2011). Myelination transition zone astrocytes are constitutively phagocytic and have synuclein dependent reactivity in glaucoma. Proc. Natl. Acad. Sci. U.S.A. 108, 1176-1181. doi: 10.1073/pnas.1013965108

Okunuki, Y., Mukai, R., Pearsall, E. A., Klokman, G., Husain, D., Park, D. H., et al. (2018). Microglia inhibit photoreceptor cell death and regulate immune cell infiltration in response to retinal detachment. Proc. Natl. Acad. Sci. U.S.A. 115, E6264-E6273.

Pankratov, Y., Lalo, U., Verkhratsky, A., and North, R. A. (2006). Vesicular release of ATP at central synapses. Pflugers Arch. 452, 589-597. doi: 10.1007/s00424006-0061-x

Pauly, D., Agarwal, D., Dana, N., Schäfer, N., Biber, J., Wunderlich, K. A., et al. (2019). Cell-Type-specific complement expression in the healthy and diseased retina. Cell Rep. 29, 2835-2848.e4.

Pease, M. E., Zack, D. J., Berlinicke, C., Bloom, K., Cone, F., Wang, Y., et al. (2009). Effect of CNTF on retinal ganglion cell survival in experimental glaucoma. Invest. Ophthalmol. Vis. Sci. 50, 2194-2200. doi: 10.1167/iovs.08-3013

Pena, J. D., Varela, H. J., Ricard, C. S., and Hernandez, M. R. (1999). Enhanced tenascin expression associated with reactive astrocytes in human optic nerve heads with primary open angle glaucoma. Exp. Eye Res. 68, 29-40. doi: 10. 1006/exer.1998.0577

Provis, J. M., Diaz, C. M., and Penfold, P. L. (1996). Microglia in human retina: a heterogeneous population with distinct ontogenies. Perspect. Dev. Neurobiol. 3 , 213-222.

Rathnasamy, G., Foulds, W. S., Ling, E. A., and Kaur, C. (2019). Retinal microglia - A key player in healthy and diseased retina. Prog. Neurobiol. 173, 18-40. doi: 10.1016/j.pneurobio.2018.05.006

Rauen, T. (2000). Diversity of glutamate transporter expression and function in the mammalian retina. Amino Acids 19, 53-62. doi: 10.1007/s007260070033

Reichenbach, A., and Bringmann, A. (2020). Glia of the human retina. Glia 68, 768-796. doi: 10.1002/glia.23727

Roche, S. L., Ruiz-Lopez, A. M., Moloney, J. N., Byrne, A. M., and Cotter, T. G. (2018). Microglial-induced Müller cell gliosis is attenuated by progesterone in a mouse model of retinitis pigmentosa. Glia 66, 295-310. doi: 10.1002/glia.23243

Rojas, B., Gallego, B. I., Ramírez, A. I., Salazar, J. J., de Hoz, R., ValienteSoriano, F. J., et al. (2014). Microglia in mouse retina contralateral to experimental glaucoma exhibit multiple signs of activation in all retinal layers. J. Neuroinflamm. 11:133. doi: 10.1186/1742-2094-11-133

Sánchez-López, A., Cuadros, M. A., Calvente, R., Tassi, M., Marín-Teva, J. L., and Navascués, J. (2004). Radial migration of developing microglial cells in quail retina: a confocal microscopy study. Glia 46, 261-273. doi: 10.1002/glia.20007

Schneider, M., and Fuchshofer, R. (2016). The role of astrocytes in optic nerve head fibrosis in glaucoma. Exp. Eye Res. 142, 49-55. doi: 10.1016/j.exer.2015.08.014

Scholz, R., Caramoy, A., Bhuckory, M. B., Rashid, K., Chen, M., Xu, H., et al. (2015). Targeting translocator protein (18 kDa) (TSPO) dampens proinflammatory microglia reactivity in the retina and protects from degeneration. J. Neuroinflamm. 12:201. 
Schuetz, E., and Thanos, S. (2004). Neuro-glial interactions in the adult rat retina after reaxotomy of ganglion cells: examination of neuron survival and phagocytic microglia using fluorescent tracers. Brain Res. Bull. 62, 391-396. doi: 10.1016/j.brainresbull.2003.10.008

Shinozaki, Y., and Koizumi, S. (2021). Potential roles of astrocytes and Müller cells in the pathogenesis of glaucoma. J. Pharmacol. Sci. 145, 262-267. doi: 10.1016/j.jphs.2020.12.009

Sierra, A., Abiega, O., Shahraz, A., and Neumann, H. (2013). Janus-faced microglia: beneficial and detrimental consequences of microglial phagocytosis. Front. Cell Neurosci. 7:6. doi: 10.3389/fncel.2013.00006

Silverman, S. M., and Wong, W. T. (2018). Microglia in the retina: roles in development, maturity, and disease. Annu. Rev. Vis. Sci. 4, 45-77. doi: 10.1146/ annurev-vision-091517-034425

Stout, C. E., Costantin, J. L., Naus, C. C., and Charles, A. C. (2002). Intercellular calcium signaling in astrocytes via ATP release through connexin hemichannels. J. Biol. Chem. 277, 10482-10488. doi: 10.1074/jbc.m109902200

Sun, D., Qu, J., and Jakobs, T. C. (2013). Reversible reactivity by optic nerve astrocytes. Glia 61, 1218-1235. doi: 10.1002/glia.22507

Supinski, G. S., Schroder, E. A., and Callahan, L. A. (2020). Mitochondria and critical illness. Chest 157, 310-322. doi: 10.1016/j.chest.2019.08.2182

Tang, Y., and Le, W. (2016). Differential roles of M1 and M2 microglia in neurodegenerative diseases. Mol. Neurobiol. 53, 1181-1194. doi: 10.1007/ s12035-014-9070-5

Tanigami, H., Okamoto, T., Yasue, Y., and Shimaoka, M. (2012). Astroglial integrins in the development and regulation of neurovascular units. Pain Res. Treat. 2012:964652.

Tezel, G., Chauhan, B. C., LeBlanc, R. P., and Wax, M. B. (2003). Immunohistochemical assessment of the glial mitogen-activated protein kinase activation in glaucoma. Invest. Ophthalmol. Vis. Sci. 44, 3025-3033. doi: $10.1167 /$ iovs.02-1136

Tezel, G., Yang, X., Luo, C., Cai, J., and Powell, D. W. (2012). An astrocyte-specific proteomic approach to inflammatory responses in experimental rat glaucoma. Invest. Ophthalmol. Vis. Sci. 53, 4220-4233. doi: 10.1167/iovs.11-9101

Thanos, S., Mey, J., and Wild, M. (1993). Treatment of the adult retina with microglia-suppressing factors retards axotomy-induced neuronal degradation and enhances axonal regeneration in vivo and in vitro. J. Neurosci. 13, 455-466. doi: 10.1523/jneurosci.13-02-00455.1993

Tribble, J. R., Harder, J. M., Williams, P. A., and John, S. W. M. (2020). Ocular hypertension suppresses homeostatic gene expression in optic nerve head microglia of DBA/2 J mice. Mol. Brain 13:81.

Uckermann, O., Wolf, A., Kutzera, F., Kalisch, F., Beck-Sickinger, A. G., Wiedemann, P., et al. (2006). Glutamate release by neurons evokes a purinergic inhibitory mechanism of osmotic glial cell swelling in the rat retina: activation by neuropeptide Y. J. Neurosci. Res. 83, 538-550. doi: 10.1002/jnr.20760

Varela, H. J., and Hernandez, M. R. (1997). Astrocyte responses in human optic nerve head with primary open-angle glaucoma. J. Glaucoma 6, 303-313.

Varnum, M. M., and Ikezu, T. (2012). The classification of microglial activation phenotypes on neurodegeneration and regeneration in Alzheimer's disease brain. Arch. Immunol. Ther. Exp. 60, 251-266. doi: 10.1007/s00005-012-0181-2

Vecino, E., Caminos, E., Ugarte, M., Martín-Zanca, D., and Osborne, N. N. (1998). Immunohistochemical distribution of neurotrophins and their receptors in the rat retina and the effects of ischemia and reperfusion. Gen. Pharmacol. 30, 305-314. doi: 10.1016/s0306-3623(97)00361-3

Vecino, E., Rodriguez, F. D., Ruzafa, N., Pereiro, X., and Sharma, S. C. (2016). Glianeuron interactions in the mammalian retina. Prog. Retin. Eye Res. 51, 1-40. doi: 10.1016/j.preteyeres.2015.06.003

Vilhardt, F., Haslund-Vinding, J., Jaquet, V., and McBean, G. (2017). Microglia antioxidant systems and redox signalling. Br. J. Pharmacol. 174, 1719-1732. doi: $10.1111 /$ bph. 13426

Wake, H., Moorhouse, A. J., Jinno, S., Kohsaka, S., and Nabekura, J. (2009). Resting microglia directly monitor the functional state of synapses in vivo and determine the fate of ischemic terminals. J. Neurosci. 29, 3974-3980. doi: 10.1523/jneurosci.4363-08.2009

Wang, K., Peng, B., and Lin, B. (2014). Fractalkine receptor regulates microglial neurotoxicity in an experimental mouse glaucoma model. Glia 62, 1943-1954. doi: $10.1002 /$ glia. 22715
Wang, M., Ma, W., Zhao, L., Fariss, R. N., and Wong, W. T. (2011). Adaptive Müller cell responses to microglial activation mediate neuroprotection and coordinate inflammation in the retina. J. Neuroinflamm. 8:173. doi: 10.1186/ 1742-2094-8-173

Wang, M., Wang, X., Zhao, L., Ma, W., Rodriguez, I. R., Fariss, R. N., et al. (2014). Macroglia-microglia interactions via TSPO signaling regulates microglial activation in the mouse retina. J. Neurosci. 34, 3793-3806. doi: 10.1523/ jneurosci.3153-13.2014

Wang, M., and Wong, W. T. (2014). Microglia-Müller cell interactions in the retina. Adv. Exp. Med. Biol. 801, 333-338. doi: 10.1007/978-1-4614-3209-8_42

Wang, Y., Chen, S., Wang, J., Liu, Y., Chen, Y., Wen, T., et al. (2021). MicroRNA-93/STAT3 signalling pathway mediates retinal microglial activation and protects retinal ganglion cells in an acute ocular hypertension model. Cell Death Dis. 12:41.

Wei, X., Cho, K. S., Thee, E. F., Jager, M. J., and Chen, D. F. (2019). Neuroinflammation and microglia in glaucoma: time for a paradigm shift. J. Neurosci. Res. 97, 70-76. doi: 10.1002/jnr.24256

Weinreb, R. N., Aung, T., and Medeiros, F. A. (2014). The pathophysiology and treatment of glaucoma: a review. JAMA 311, 1901-1911. doi: 10.1001/jama. 2014.3192

Weinreb, R. N., and Khaw, P. T. (2004). Primary open-angle glaucoma. Lancet 363 , $1711-1720$.

West, H., Richardson, W. D., and Fruttiger, M. (2005). Stabilization of the retinal vascular network by reciprocal feedback between blood vessels and astrocytes. Development 132, 1855-1862. doi: 10.1242/dev.01732

Wheeler, M. A., Clark, I. C., Tjon, E. C., Li, Z., Zandee, S. E. J., Couturier, C. P., et al. (2020). MAFG-driven astrocytes promote CNS inflammation. Nature 578, 593-599.

Wiemann, S., Reinhard, J., Reinehr, S., Cibir, Z., Joachim, S. C., and Faissner, A. (2020). Loss of the extracellular matrix molecule tenascin-C Leads to absence of reactive gliosis and promotes anti-inflammatory cytokine expression in an autoimmune glaucoma mouse model. Front. Immunol. 11:566279. doi: 10.3389/ fimmu.2020.566279

Williams, P. A., Marsh-Armstrong, N., Howell, G. R., and Lasker/IRRF Initiative on Astrocytes and Glaucomatous Neurodegeneration Participants (2017). Neuroinflammation in glaucoma: a new opportunity. Exp. Eye Res. 157, 20-27.

Xue, W., Cojocaru, R. I., Dudley, V. J., Brooks, M., Swaroop, A., and Sarthy, V. P. (2011). Ciliary neurotrophic factor induces genes associated with inflammation and gliosis in the retina: a gene profiling study of flow-sorted, Müller cells. PLoS One 6:e20326. doi: 10.1371/journal.pone.0020326

Yan, L., Lee, S., Lazzaro, D. R., Aranda, J., Grant, M. B., Chaqour, B., et al. (2015). Single and compound knock-outs of MicroRNA (miRNA)-155 and its angiogenic gene target $\mathrm{CCN} 1$ in Mice alter vascular and neovascular growth in the retina via resident Microglia. J. Biol. Chem. 290, 23264-23281.

Yuan, L., and Neufeld, A. H. (2001). Activated microglia in the human glaucomatous optic nerve head. J. Neurosci. Res. 64, 523-532.

Zhang, X., Cheng, M., and Chintala, S. K. (2004). Kainic acid-mediated upregulation of matrix metalloproteinase-9 promotes retinal degeneration. Invest. Ophthalmol. Vis. Sci. 45, 2374-2383.

Zhao, X. F., Wan, J., Powell, C., Ramachandran, R., Myers, M. G. Jr., and Goldman, D. (2014). Leptin and IL-6 family cytokines synergize to stimulate Müller glia reprogramming and retina regeneration. Cell Rep. 9, 272-284.

Zhu, K., Zhang, M. L., Liu, S. T., Li, X. Y., Zhong, S. M., Li, F., et al. (2017). Ghrelin attenuates retinal neuronal Autophagy and apoptosis in an experimental rat glaucoma model. Invest. Ophthalmol. Vis. Sci. 58, 6113-6122.

Conflict of Interest: The authors declare that the research was conducted in the absence of any commercial or financial relationships that could be construed as a potential conflict of interest.

Copyright (c) 2021 Zhao, Sun, Luo, Wang and Sun. This is an open-access article distributed under the terms of the Creative Commons Attribution License (CC BY). The use, distribution or reproduction in other forums is permitted, provided the original author(s) and the copyright owner(s) are credited and that the original publication in this journal is cited, in accordance with accepted academic practice. No use, distribution or reproduction is permitted which does not comply with these terms. 